enough to recapitulate for an examination answer, but practically of little value.

In urology there are still some gaps in descriptions of operative work readily available to the postgraduate student and these specialist textbooks are therefore to be welcomed. Swinney and Hammersley's 'Handbook' achieves just the required level of explicit instruction throughout most of its considerable range, including valuable details of technique, materials used and manoeuvres to overcome common difficulties. The reader senses the guiding hand of an experienced teacher and texts and illustrations generally give a clear idea of procedure.

To be particularly critical, it is a little irritating that even though the artist is here co-author on occasions the illustrations are not altogether in accord with the text, as for example, under nephrectomy where the ureter is shown tied at the ureteric junction.

The book is indeed comprehensive and the authors disarm the critic in the preface by claiming to present the description only of those operations that they have found of most use in practice; however, with the increased interest in operations on the renal artery a fuller description of the abdominal transperitoneal approach to the kidney would doubtless be welcomed by many readers, who would surely turn to a volume like this for just such help. For the operation of partial nephrectomy no comment is made of any cooling procedure which might be a valuable addition. These points are mentioned perhaps less as criticisms than in the hope that these additions may be made in future editions of the volume, which can be recommended as giving just the sort of clear and practical help which we are looking for in a book of operative surgery. For the same reason Kyril Conger's monograph on Transurethral Prostatic Surgery must also be commended. It of course covers quite a narrow field, virtually, one endoscopic procedure. It does so with such painstaking care, however, and such thought for the smallest detail that there must be something of value in it for any urologist. The author is clearly familiar with the whole literature of endoscopic work and the management of its complications and his book gives a good summary of these as well as valuable personal opinions based on wide experience. There is no point in drawing attention to some of the well known differences between practice in the United States and this Country, such as testing residual urine by the passage of a catheter. The text as it stands is a most complete and readable guide which teaches, perhaps, as much as can be imparted about a complex technical task on a printed page.

\section{Cancer-A General Guide to Research and its Treatment}

Editor N. N. Petrov, translated A. P. Fletcher. Pp. xvi +387 , illustrated. Oxford, London, New York and Paris: Pergamon Press. 1962. 80 s.

Western visitors to the Soviet Union are frequently struck by unfamiliar contrasts between the ultra-modern and the curiously old fashioned-jets on the tarmac and straw country brushes to sweep the passenger lounge or the splendid contemporary Kremlin Palace of Congresses beside the usual draped, plush-covered interiors everywhere else.

The present volume, edited and partly wirtten by a distinguished Soviet oncologist together. with a team of well-known collaborators, sets out to provide medical practitioners and senior students with a general guide to the field of cancer covering theoretical considerations, the huge subject of experimental research, as well as many aspects of therapy.

The treatment is wide and the historical and classical pathological aspects are admirably dealt with. But in other fields there is the faint but unmistakable flavour of the Victorian mingling with the modern, so typical of things Russian outside rocketry and space travel. Current ideas are indeed mentioned but are lost to view in a mass of out-of-date considerations.

The immense strides currently being made in our knowledge of both normal and pathological cell behaviour and the contribution they have made to the understanding of cancer causation-particularly in regard to the role of viruses, changes in the surface properties of cells, and the mode of action of carcinogenic chemicalsare largely disregarded by the Russian authors. References a dozen years old are termed 'recent' in this fastmoving field even though the book was published in Leningrad as late as 196r. Modern views on cancer and the latest developments in cancer research are not adequately described.

On the clinical side, the approach is rather simple and might serve for students and perhaps for practitioners in a society whose medical services are developing, but for Western medical men the treatment is too unsophisticated to be of great value.

The book has been fairly well translated, is very expensive, and can really only be recommended for the insight it gives into the views and attitudes of our Soviet colleagues on one of the more important problems of medical science today.

\section{Clinical Physiology}

Edited by E. J. M. Campbell, C. J. Dickinson and J. D. H. Slater. 2nd Edition. Pp. xiii +605 , illustrated. Oxford: Blackwell. r963. 57s. $6 d$.

A second edition of this popular book has now appeared. There has been detailed revision with preservation of the basic design of each chapter. There are two new chapters; one on energy sources and utilization incorporates the previous section on the regulation of blood sugar and an entirely new section on the nervous system in the control of movement has been written by Dr. A. J. Buller. The value of the book has been increased by these changes and by the care with which new material has been added to all sections; it is surprising how much 'physiology' has contributed to clinical medicine in the three years since the first edition.

\section{Modern Concepts of the Pathogenesis of Coronary Atherosclerosis}

A. Whitley Branwood. Pp. vii + r44, illustrated. Edinburgh \& London: E. \& S. Livingstone. 1963. $30 s$.

In this small book the author sets out 'to bring together certain important aspects of the many factors concerned in the pathogenesis of atherosclerosis'. The most valuable sections are however those which deal with the author's present field, which is the behaviour of the arterial intima in tissue culture. Many of the other chapters are extremely short, which gives the book a fragmentary pattern, and the author has tended to accept rather uncritically some of the work which he quotes. He lays great stress on the difference between 'hard' and 'soft' arterial plaques, and goes on to suggest that this difference might have significant implications in the treatment of patients with arterial disease. Other workers however, feel that there is no real evidence as yet that the differences indicate two distinct types of plaque, for they could be the two extreme forms of a continuous spectrum of plaque characteristics.

Unhappily the book is full of errors; first, in simple spelling (anticoagulent; coaguability; polyethmoid fatty acids); second, in the references (Virchow's collected 